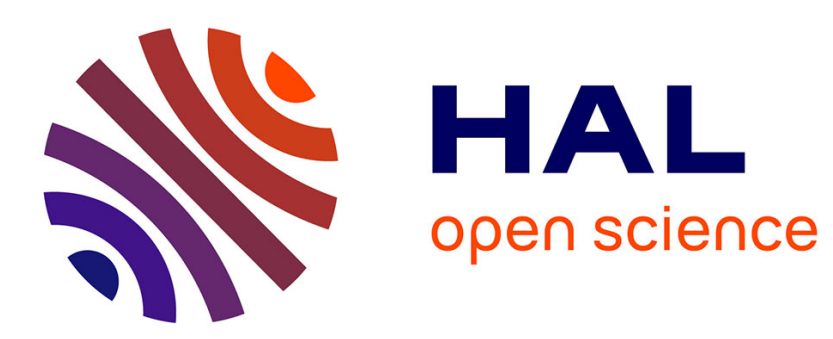

\title{
Zeta Functions and Monodromy for Surfaces that are General for a Toric Idealistic Cluster
}

\author{
Ann Lemahieu, Willem Veys
}

\section{To cite this version:}

Ann Lemahieu, Willem Veys. Zeta Functions and Monodromy for Surfaces that are General for a Toric Idealistic Cluster. International Mathematics Research Notices, 2009, 9 (1), pp.11-62. 10.1093/imrn/rnn122 . hal-01279765

\section{HAL Id: hal-01279765 https://hal.science/hal-01279765}

Submitted on 26 May 2016

HAL is a multi-disciplinary open access archive for the deposit and dissemination of scientific research documents, whether they are published or not. The documents may come from teaching and research institutions in France or abroad, or from public or private research centers.
L'archive ouverte pluridisciplinaire HAL, est destinée au dépôt et à la diffusion de documents scientifiques de niveau recherche, publiés ou non, émanant des établissements d'enseignement et de recherche français ou étrangers, des laboratoires publics ou privés. 


\title{
Zeta functions and monodromy for surfaces that are general for a toric idealistic cluster
}

\author{
Ann Lemahieu and Willem Veys*
}

\begin{abstract}
In this article we consider surfaces that are general with respect to a 3dimensional toric idealistic cluster. In particular, this means that blowing up a toric constellation provides an embedded resolution of singularities for these surfaces. First we give a formula for the topological zeta function directly in terms of the cluster. Then we study the eigenvalues of monodromy. In particular, we derive a useful criterion to be an eigenvalue. In a third part we prove the monodromy and the holomorphy conjecture for these surfaces.
\end{abstract}

\section{Contents}

1. Introduction 1

2. Toric clusters 4

3. Conjectures 10

4. Computation of the topological zeta function 12

5. Analysis of $\chi\left(E_{i}^{\circ}\right) \quad 16$

6. Determination of the sign of $\chi\left(E_{i}^{\circ}\right) \quad 24$

7. The monodromy conjecture for candidate poles of order 1

8. The monodromy conjecture for candidate poles of order 2 or $3 \quad 35$

9. The holomorphy conjecture 41

\section{INTRODUCTION}

In [We65], Weil introduced some zeta functions $\mathcal{Z}(K, f)$ that are integrals over a $p$-adic field $K$ and that are associated to a polynomial $f(\underline{x}) \in K[\underline{x}]$. Using embedded resolution of singularities, Igusa showed that these zeta functions are rational and he studied their poles (see $[\operatorname{Ig} 75]$ and $[\operatorname{Ig} 78]$ ). One can define the analogous integrals over $K=\mathbb{R}$ or $\mathbb{C}$. Also these zeta functions are rational (see for example [At70] and [BeGe69]) and it is known that their poles are contained in the set of roots - and roots shifted by a negative integer - of the Bernstein polynomial $b_{f}$. By Malgrange ([Ma83]), if $\alpha$ is a root of $b_{f}$, then $e^{2 \pi i \alpha}$

*Ann Lemahieu, Willem Veys, K.U.Leuven, Departement Wiskunde, Celestijnenlaan 200B, B-3001 Leuven, Belgium, email: lemahieu@mathematik.uni-kl.de, wim.veys@wis.kuleuven.ac.be. The research was partially supported by the Fund of Scientific Research - Flanders (G.0318.06) and MEC PN I+D+I MTM2007-64704. 
is an eigenvalue of the local monodromy of $f$ at some point of $f^{-1}(0)$. So when $K=\mathbb{R}$ or $\mathbb{C}$, then the poles of the zeta function induce eigenvalues of the local monodromy. This result was a motivation to study this relation at the $p$-adic side. The study of concrete examples made it natural to propose the following conjecture.

Monodromy conjecture. ([Ig88]) Let $F \subset \mathbb{C}$ be a number field and $f \in F[\underline{x}]$. For almost all $p$-adic completions $K$ of $F$, if $s_{0}$ is a pole of $\mathcal{Z}(K, f)$, then $e^{2 \pi i R e\left(s_{0}\right)}$ is an eigenvalue of the local monodromy of $f$ at some point of the hypersurface $f=0$.

Loeser verified this conjecture for plane curves (see [L88]). He also gave a proof for a class of polynomials in higher dimensions; the polynomial should be nondegenerate with respect to its Newton polyhedron and should satify some numerical conditions ([L90] and Section 3).

When Denef and Loeser introduced the topological zeta function in 1992 in [De,L92], an analogous version of the monodromy conjecture arose. This monodromy conjecture relates the poles of the topological zeta function $Z_{t o p, f}$ associated to a polynomial function or a germ of a holomorphic function $f$ with the eigenvalues of monodromy of the hypersurface $f=0$.

Monodromy conjecture. If $s_{0}$ is a pole of $Z_{t o p, f}$, then $e^{2 \pi i s_{0}}$ is an eigenvalue of the local monodromy of $f$ at some point of the hypersurface $f=0$.

By the original definition of the topological zeta function, it follows that the monodromy conjecture for the Igusa zeta function implies the monodromy conjecture for the topological zeta function. Artal Bartolo, Cassou-Noguès, Luengo and Melle Hernández proved the monodromy conjecture for some surface singularities, such as the superisolated ones (see [A-B,C-N,Lu,M-H02]), and for quasi-ordinary polynomials in $[\mathrm{A}-\mathrm{B}, \mathrm{C}-\mathrm{N}, \mathrm{Lu}, \mathrm{M}-\mathrm{H} 06]$. The second author provided results in [Ve93B], [Ve95] and [Ve06], and together with Rodrigues in [RVe03]. In [LeVe07], the authors consider the same context as in this paper but they had to impose a restricting condition on the surfaces. Via geometrical arguments they showed that the monodromy conjecture holds for candidate poles of the topological zeta function of order 1 that are poles.

There are more conjectures relating the poles of the topological zeta function (Igusa zeta function) and the eigenvalues of monodromy. There exist the rational functions $Z_{t o p, f}^{(r)}\left(r \in \mathbb{Z}_{>0}\right)$ that are variants of the topological zeta function and that play a role in the holomorphy conjecture, which was stated by Denef.

Holomorphy conjecture. ([De91]) If $r \in \mathbb{Z}_{>0}$ does not divide the order of any eigenvalue of the local monodromy of $f$ at any point of $f^{-1}\{0\}$, then $Z_{\text {top }, f}^{(r)}$ is holomorphic on $\mathbb{C}$.

Originally the holomorphy conjecture was formulated for the Igusa zeta func- 
tion. We refer to [De91] for the inspiration. Denef showed that the conjecture is true for the relative invariants of a few prehomogeneous vector spaces. The second author proved the conjecture for plane curves (see [Ve93A]) and together with Rodrigues for homogeneous polynomials (see [RVe01]).

Although the monodromy conjecture and/or holomorphy conjecture has been proven for these kinds of singularities, one did not get a better understanding of the deep reason why the conjectures hold for them. Until now, the attempts are thus restricted to prove the conjecture for classes of singularities.

This article deals with the class of surfaces that are general with respect to a 3-dimensional toric idealistic cluster. This implies that we work with surfaces for which there exists an embedded resolution of singularities by blowing up in points that are orbits for the action of the torus, i.e. in a toric constellation. We refer to Section 2 for a recap about clusters and in Section 3 we explain the objects that play the main role in the conjecture. In Section 4 we show how the topological zeta function can be computed directly in terms of the toric cluster for the surfaces that we consider. We use the embedded resolution provided by the blowing up of the constellation. Let $\pi: Z \rightarrow \mathbb{C}^{3}$ be that resolution of such a surface $f=0$ and let $E_{j}, j \in S$, be the irreducible components obtained by this resolution of which $E_{1}, \cdots, E_{r}$ are the exceptional ones. We will denote $E_{j}^{\circ}:=E_{j} \backslash\left(\cup_{i \in S \backslash\{j\}} E_{i}\right)$, for $j \in S$. We write $N_{j}$ and $\nu_{j}-1$ for the multiplicities of $E_{j}$ in the divisor on $Z$ of $f \circ \pi$ and $\pi^{*}(d x \wedge d y \wedge d z)$, respectively. The numbers $-\nu_{j} / N_{j}, j \in S$, form a complete list of candidate poles of $Z_{t o p, f}$.

We compute in particular the Euler characteristic of the spaces $E_{j}^{\circ}, 1 \leq j \leq$ $r$, in terms of the cluster. They show up in A'Campo's formula for the eigenvalues of monodromy and they are very relevant for the monodromy conjecture. In a fifth section we analyse these Euler characteristics. Our goal is to determine when these numbers are less than or equal to 0. A geometric argument will show that we can reduce this job to the investigation of a finite number of families of constellations. We complete Section 5 with combinatorial preparations. These make it possible to determine the sign of the Euler characteristics that we are looking for. We carry this out in Section 6. We then prove the following result.

Theorem. If $\chi\left(E_{j}^{\circ}\right)>0$, then $e^{-2 \pi i \frac{\nu_{j}}{N_{j}}}$ is an eigenvalue of monodromy of $f$.

Using this result, we prove in Section 7 the monodromy conjecture for candidate poles of order 1 that are poles and in Section 8 the monodromy conjecture for candidate poles of order 2 or 3 that are poles. Hence, we obtain:

Theorem. Let $f$ be a germ of a polynomial map that is general with respect to a 3 -dimensional toric idealistic cluster. If $s_{0}$ is a pole of $Z_{t o p, f}$, then $e^{2 \pi i s_{0}}$ is an eigenvalue of monodromy of $f$ at some point of the hypersurface $f=0$. 
In Section 9 we prove the holomorphy conjecture for these surfaces.

Theorem. Let $f$ be a germ of a polynomial map that is general with respect to a 3 -dimensional toric idealistic cluster. If $r \in \mathbb{Z}_{>0}$ does not divide the order of any eigenvalue of the local monodromy of $f$ at any point of $f=0$, then $Z_{t o p, f}^{(r)}$ is holomorphic on $\mathbb{C}$.

\section{TORIC CLUSTERS}

In this section we introduce the terminology of infinitely near points, (toric) clusters etc. according to [C,G-S,L-J96]. We would like to refer to [C,G-S,L-J96] for some historical notes on clusters. See also [Ca90], [Ca00], [En,Ch15], [Li69], [Li78], [Li94] and [Z38] for more details on the theory of clusters.

2.1. Clusters. - Let $X$ be a nonsingular variety of dimension $d \geq 2$ and let $Z$ be a variety obtained from $X$ by a finite succession of point blowing-ups. A point $Q \in Z$ is said to be infinitely near to a point $P \in X$ if $P$ is in the image of $Q$; we write $Q \geq P$. A constellation is a finite sequence $\mathcal{C}:=\left\{Q_{1}, Q_{2}, \cdots, Q_{r}\right\}$ of infinitely near points of $X$ with $Q_{1} \in X=: X_{0}$ and each $Q_{j+1}$ is a point on the variety $X_{j}$ obtained by blowing up $Q_{j}$ in $X_{j-1}, j \in\{1, \cdots, r-1\}$. The variety $X(\mathcal{C}):=X_{r}$ obtained by blowing up $Q_{r}$ in $X_{r-1}$ is called the sky.

The relation ' $\geq$ ' gives rise to a partial ordering on the points of a constellation. In the case that they are totally ordered, so $Q_{r} \geq \cdots \geq Q_{1}$, the constellation $\mathcal{C}$ is called a chain. For every $Q_{j}$ in $\mathcal{C}$, the subsequence $\mathcal{C}^{j}:=\left\{Q_{i} \mid Q_{j} \geq Q_{i}\right\}$ of $\mathcal{C}$ is a chain. The integer $l\left(Q_{j}\right):=\# \mathcal{C}^{j}-1$ is called the level of $Q_{j}$. In particular $Q_{1}$ has level 0 . If no other point of $\mathcal{C}$ has level 0 then $Q_{1}$ is called the origin of $\mathcal{C}$. We will always work with constellations that have an origin and we will also denote the origin of the constellation by $o$. If $Q_{j} \geq Q_{i}$ and $l\left(Q_{j}\right)=l\left(Q_{i}\right)+1$, we will write $Q_{j} \succ Q_{i}$ or $j \succ i$.

For each $Q_{i} \in \mathcal{C}$, denote the exceptional divisor of the blowing-up in $Q_{i}$ by $E_{i}$, as well as its strict transform at some intermediate stage (including the final stage) $X_{j}, i \leq j \leq r$. The total transform at some intermediate stage (including the final stage) will be denoted by $E_{i}^{*}$. If $Q_{j} \in E_{i}$, then one says that $Q_{j}$ is proximate to $Q_{i}$. This will be denoted as $Q_{j} \rightarrow Q_{i}$ or $j \rightarrow i$. As $E_{i}=E_{i}^{*}-\sum_{j \rightarrow i} E_{j}^{*}$, it follows that also $\left\{E_{1}^{*}, \cdots, E_{r}^{*}\right\}$ is a basis of the group of divisors with exceptional support $\oplus_{j=1}^{r} \mathbb{Z} E_{j}$.

A pair $\mathcal{A}:=(\mathcal{C}, \underline{m})$ consisting of a constellation $\mathcal{C}:=\left\{Q_{1}, \cdots, Q_{r}\right\}$ and a sequence $\underline{m}:=\left(m_{1}, \cdots, m_{r}\right)$ of nonnegative integers is called a cluster. One calls $m_{j}$ the weight or multiplicity of $Q_{j}$ in the cluster and we write $D(\mathcal{A}):=\sum_{j=1}^{r} m_{j} E_{j}^{*}$. Introducing the numbers $v_{j}, 1 \leq j \leq r$, by setting $m_{j}:=v_{j}-\sum_{j \rightarrow i} v_{i}$, allows us to write also $D(\mathcal{A})=\sum_{j=1}^{r} v_{j} E_{j}$.

The idea of clusters is to express that a system of hypersurfaces is passing through the points of the constellation with (at least) the given multiplicities. Blowing up a point $Q_{i} \in \mathcal{C}$ induces a discrete valuation $\nu_{i}$ on $\mathbb{C}(X) \backslash\{0\}$ : for 
$g \in \mathbb{C}(X) \backslash\{0\}$, the value $\nu_{i}(g)$ is the order of the pullback of $g$ (at the stage $X_{i}$ ) along $E_{i}$. To a cluster we can then associate the (complete) ideal

$$
I\left(v_{1}, \cdots, v_{r}\right)=\left\{g \in \mathcal{O}_{X, o} \mid \nu_{j}(g) \geq v_{j}, 1 \leq j \leq r\right\} \cup\{0\} .
$$

If we want that these ideals principalise by blowing up the points of the constellation, we require the ideals to be finitely supported. Formally, an ideal $I$ in $\mathcal{O}_{X, o}$ is called finitely supported if $I$ is primary for the maximal ideal $\mathrm{m}$ of $\mathcal{O}_{X, o}$ - so supported at the closed point - and if there exists a constellation $\mathcal{C}$ of infinitely near points of $X$ such that $I \mathcal{O}_{X(\mathcal{C})}$ is an invertible sheaf.

On the other hand, given a finitely supported ideal $I$, one can associate a cluster to it. Let $\mathcal{C}_{I}=:\left\{Q_{1}, \cdots, Q_{r}\right\}$ be the constellation of base points of $I$, i.e. the minimal constellation $\mathcal{C}$ such that $I \mathcal{O}_{X(\mathcal{C})}$ is an invertible sheaf. Let $m_{j}$ be the order of the point $Q_{j}, 1 \leq j \leq r$ in the strict transform of the ideal $I$ in $\mathcal{O}_{X_{j}, Q_{j}}$. Then the ideal sheaf $I \mathcal{O}_{X\left(\mathcal{C}_{I}\right)}$ is associated to $-D\left(\mathcal{A}_{I}\right):=$ $-\sum_{j=1}^{r} m_{j} E_{j}^{*}$.

If $\mathcal{C}$ is a constellation with origin at $Q_{1}$, the cluster $\mathcal{A}:=(\mathcal{C}, \underline{m})$ is called idealistic if there exists a finitely supported ideal $I$ in $\mathcal{O}_{X, Q_{1}}$ such that $I \mathcal{O}_{X(\mathcal{C})}$ is the ideal sheaf associated to $-D(\mathcal{A})$. For an idealistic cluster $\mathcal{A}$, Lipman proved that there exists a unique finitely supported complete ideal $I_{\mathcal{A}}$ such that $I_{\mathcal{A}} \mathcal{O}_{X(\mathcal{C})}=\mathcal{O}_{X(\mathcal{C})}(-D(\mathcal{A}))$, namely that given by the direct image of $\mathcal{O}_{X(\mathcal{C})}(-D(\mathcal{A}))$ in $X$, see $[\mathrm{Li} 78]$.

In the next subsection we will illustrate these notions in the context of our results.

2.2. Toric clusters in $\mathbb{C}^{3}$.- From now on suppose that $X$ is the affine toric variety $\mathbb{C}^{3}$. Let $Q_{1}$ be the origin of $\mathbb{C}^{3}=X_{0}$. A 3-dimensional toric constellation of infinitely near points with origin $Q_{1}$ is a constellation $\mathcal{C}:=\left\{Q_{1}, Q_{2}, \cdots, Q_{r}\right\}$ such that each $Q_{j+1}$ is a 0 -dimensional orbit in the toric variety $X_{j}$ obtained by blowing up $Q_{j}$ in $X_{j-1}, 1 \leq j \leq r-1$. Blowing up in orbits of smooth varieties corresponds to making star subdivisions of the fan corresponding to the variety (see for example [O78]). In this way each blowing-up in a 0-dimensional orbit induces the creation of three cones of dimension 3 and thus of three new 0 -dimensional orbits. Hence, the choice of a point $Q_{i}$ in a toric chain is equivalent to the choice of an integer $a_{i} \in\{1,2,3\}$, which determines a 3 -dimensional cone in the fan. A tree with a root such that each vertex has at most three following adjacent vertices is called a 3-nary tree. The above observation shows that there is a natural bijection between the set of 3-dimensional toric constellations with origin and the set of finite 3 -nary trees with a root, with the edges labeled with positive integers not greater than 3 , such that two edges with the same source have different labels.

A cluster $\mathcal{A}:=(\mathcal{C}, \underline{m})$ is called toric if the constellation $\mathcal{C}$ is toric. The blowing-ups now induce monomial valuations (i.e. valuations determined by their values on monomials) and the ideal $I\left(v_{1}, \cdots, v_{r}\right)$ associated to a toric cluster is thus monomial. 


\section{Example 1}

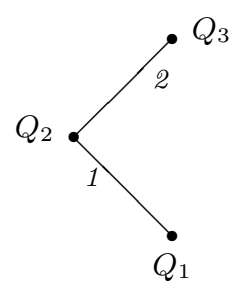

Suppose $d=3$ and $\mathcal{C}$ is the constellation pictured at the left. It represents the following resolution process: by blowing up in the origin $Q_{1}$ we get an exceptional variety $E_{1} \cong \mathbb{P}^{2}$. In $E_{1}$ there is one point in which we blow up, namely $Q_{2}$. The labels indicate in which affine chart the points of the constellation are created.

We call the affine chart with label 1 that one in which the equation of $E_{1}$ is $x=0$. In the chart with label 2 one has $E_{1} \leftrightarrow y=0$ and in the chart with label 3 one has $E_{1} \leftrightarrow z=0$. The point $Q_{2}$ is the origin of the affine chart with label 1. After blowing up in $Q_{2}$ we get an exceptional variety $E_{2} \cong \mathbb{P}^{2}$, where we blow up in the point $Q_{3}$ that is the origin of chart 1.2. There one has $E_{2} \leftrightarrow y=0$ and (the transform of) $E_{1} \leftrightarrow x=0$.

We now point out how the induced valuations $\nu_{1}, \nu_{2}$ and $\nu_{3}$ act. For $a, b, c \in \mathbb{Z}_{>0}$, $\nu_{1}\left(x^{a} y^{b} z^{c}\right)=a+b+c$ because the pullback of $x^{a} y^{b} z^{c}$ in chart 1 is $x^{a+b+c} y^{b} z^{c}$. The pullback in chart 1.2 becomes $x^{a+b+c} y^{a+2 b+2 c} z^{c}$ and thus $\nu_{2}\left(x^{a} y^{b} z^{c}\right)=$ $a+2 b+2 c$. Analogously we find $\nu_{3}\left(x^{a} y^{b} z^{c}\right)=2 a+3 b+4 c$. We can represent these valuations by the following vectors in the lattice $\mathbb{N}^{3}$ :

$$
\nu_{1} \leftrightarrow(1,1,1) \quad \nu_{2} \leftrightarrow(1,2,2) \quad \nu_{3} \leftrightarrow(2,3,4)
$$

We have $I\left(v_{1}, v_{2}, v_{3}\right)=\left(x^{a} y^{b} z^{c} \mid a+b+c \geq v_{1}, a+2 b+2 c \geq v_{2}, 2 a+3 b+\right.$ $\left.4 c \geq v_{3}\right)$. To compute such an ideal, one can picture the hyperplanes induced by the valuations. We compute this ideal for the cluster $\mathcal{A}:=(\mathcal{C}, \underline{m})$ with $\left(m_{1}, m_{2}, m_{3}\right)=(2,1,1)$. Then $\left(v_{1}, v_{2}, v_{3}\right)=(2,3,6)$ and one can see from

that $I(2,3,6)=\left(x^{3}, y^{2}, z^{2}, x z, x^{2} y, y z\right)$. One can verify that this ideal is finitely supported and that the cluster associated to this ideal is exactly the cluster $\mathcal{A}$. Hence this cluster is idealistic.

Let us now consider the cluster $\mathcal{B}$ consisting of the above constellation and for which $\left(m_{1}, m_{2}, m_{3}\right)=(4,1,2)$ or $\left(v_{1}, v_{2}, v_{3}\right)=(4,5,11)$. Analogously one finds that $I:=I(4,5,11)$ is equal to

$$
\left(x^{6}, y^{4}, z^{4}, x y^{3}, x^{3} y^{2}, x^{4} y, x^{3} z^{2}, x^{4} z, x z^{3}, y z^{3}, y^{2} z^{2}, y^{3} z, x y z^{2}, x y^{2} z, x^{2} y z\right) .
$$

One finds that this ideal is finitely supported but the cluster associated to this ideal is

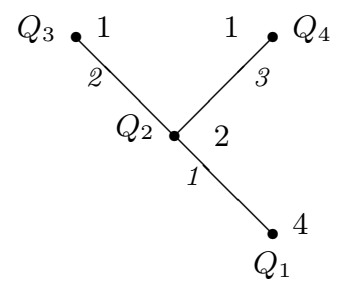


From this we can deduce that $\mathcal{B}$ is not an idealistic cluster. Indeed, if $\mathcal{B}$ was idealistic, then there would exist a finitely supported complete ideal $J$ such that $J \mathcal{O}_{X(\mathcal{C})}=\mathcal{O}_{X(\mathcal{C})}(-D(\mathcal{B}))$ and we also know that $J$ would be included in $I$. As $I$ and $J$ are both complete ideals, they should be equal but we mentioned already that $I \mathcal{O}_{X(\mathcal{C})} \neq \mathcal{O}_{X(\mathcal{C})}(-D(\mathcal{B}))$.

Finally let us consider the cluster consisting of the constellation $\mathcal{C}$ with $\left(m_{1}, m_{2}, m_{3}\right)=(2,2,2)$ or $\left(v_{1}, v_{2}, v_{3}\right)=(2,4,8)$. One can check that

$$
I(2,4,8)=\left(x^{4}, y^{3}, z^{2}, x y^{2}, x^{3} y, x^{2} z, y^{2} z, x y z\right)
$$

and that this ideal is even not finitely supported. In the same way we can conclude that this cluster is not idealistic.

2.3. Properties.- In this subsection we recall some properties about clusters, in particular about toric clusters.

(1) In the case of toric clusters, there exists a combinatorial characterisation for the idealistic clusters. Fix a point $Q_{i}$ in a toric 3-dimensional constellation $\mathcal{C}$ and some integers $a, b$ such that $a, b \in\{1,2,3\}$ and $a \neq b$. For $s, t \in \mathbb{Z}_{\geq 0}$, let $Q_{i}\left(a^{s}, b^{t}\right)$ be the terminal point of the chain with origin $Q_{i}$ coded by $(a, \cdots, a, b, \cdots, b)$ where $a$ appears $s$ times and $b$ appears $t$ times. If $t=0$, it is denoted by $Q_{i}\left(a^{s}\right)$. The point $Q_{i}\left(a^{s}, b^{t}\right)$ may not belong to $\mathcal{C}$. A point $Q_{j} \in \mathcal{C}$ that is infinitely near to $Q_{i}$ is said to be linearly proximate to $Q_{i}$, if $Q_{j}=Q_{i}\left(a, b^{t}\right)$, with $a, b$ and $t$ as above. We denote this relation by $Q_{j} \rightarrow Q_{i}$ or $j \rightarrow i$. Then we have that $Q_{j}$ is linearly proximate to $Q_{i}$ if and only if there exists a 1-dimensional orbit I in $B_{i}$ such that $Q_{j}$ belongs to the strict transform of the closure of $I$ in $E_{i}$. This explains the terminology. Denote $M_{Q_{i}}(a, b):=\sum_{t \geq 0} m_{Q_{i}\left(a, b^{t}\right)}$. Campillo, Gonzalez-Sprinberg and Lejeune-Jalabert show the following.

1. A toric cluster $\mathcal{A}=(\mathcal{C}, \underline{m})$ is idealistic if and only if for each point $Q_{i}$ of the constellation $\mathcal{C}$ and for each pair of integers $a$ and $b$ such that $a, b \in\{1,2,3\}$ and $a \neq b$, the following inequality is satisfied:

$$
M_{Q_{i}}(a, b)+M_{Q_{i}}(b, a) \leq m_{Q_{i}} .
$$

These inequalities are called the linear proximity inequalities.

2. Let $\mathcal{A}=(\mathcal{C}, \underline{m})$ be a 3 -dimensional toric idealistic cluster with associated divisor $D(\mathcal{A})=\sum_{j=1}^{r} m_{j} E_{j}^{*}=\sum_{j=1}^{r} v_{j} E_{j}$ and let $\nu_{1}, \cdots, \nu_{r}$ be the induced discrete valuations. Such a valuation is called Rees for the ideal $I(\underline{v}):=I\left(v_{1}, \cdots, v_{r}\right)$ if it is a valuation induced by an irreducible component of the exceptional divisor of the normalised blowing-up $\overline{B l_{I(\underline{v})} X}$ of $I(\underline{v})$. Then

$$
\forall Q_{i} \in \mathcal{C}: m_{i}^{2} \geq \sum_{j \rightarrow i} m_{j}^{2} \quad \text { and }
$$

$$
\nu_{i} \text { is Rees for } I(\underline{v}) \text { if and only if } m_{i}^{2}>\sum_{j \rightarrow i} m_{j}^{2} .
$$


(2) To a monomial ideal $I$ one can associate a Newton polyhedron $\mathcal{N}_{I}$. It is the convex hull of $m+\mathbb{R}_{\geq 0}^{3}$ as $m$ runs through the set of exponents of monomials in $I$. We refer to $[\mathrm{Ke}, \mathrm{Kn}, \mathrm{M}, \mathrm{S} 73]$ for the proofs of the following properties.

1. The compact facets of $\mathcal{N}_{I}$ correspond with the Rees valuations of $I$.

2. A monomial ideal is complete if and only if it contains every monomial whose exponent is a point of $\mathcal{N}_{I} \cap \mathbb{Z}_{\geq 0}^{3}$.

(3) Campillo, Gonzalez-Sprinberg and Lejeune-Jalabert generate a very interesting set of 'general' hypersurfaces in [C,G-S,L-J96]:

Theorem 1 The canonical map from the sky of the constellation of base points of a finitely supported ideal $I$ to $X$ is an embedded resolution of the subvariety of $(X, o)$ defined by a general enough element in $I$.

We will call these 'general enough' elements general for $I$ or general for $\mathcal{C}_{I}$. We will prove the monodromy and holomorphy conjectures for the class of surfaces that are general for a finitely supported monomial ideal. In particular this means that our results apply to all surfaces for which there exists an embedded resolution consisting of toric point blowing ups and for which the corresponding toric cluster is idealistic. According to Theorem 1 such surfaces in a finitely supported ideal form an open dense set.

\section{Example 2}

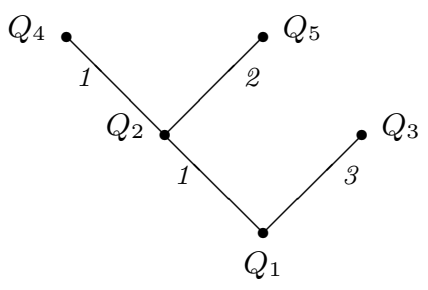

Suppose $d=3$ and $\mathcal{C}$ is the constellation pictured at the left. It represents the following resolution process: by blowing up in the origin $Q_{1}$ we get an exceptional variety $E_{1} \cong \mathbb{P}^{2}$. In $E_{1}$ there are two points in which we blow up, namely $Q_{2}$ and $Q_{3}$. After blowing up in $Q_{2}$ we get an exceptional variety $E_{2} \cong \mathbb{P}^{2}$, where again we blow up in two points.

The induced valuations are represented by the following vectors in the lattice $\mathbb{N}^{3}$ :

$\nu_{1} \leftrightarrow(1,1,1) \quad \nu_{2} \leftrightarrow(1,2,2) \quad \nu_{3} \leftrightarrow(2,2,1) \quad \nu_{4} \leftrightarrow(1,3,3) \quad \nu_{5} \leftrightarrow(2,3,4)$

Consider the multiplicities $\left(m_{1}, m_{2}, m_{3}, m_{4}, m_{5}\right)=(3,2,1,1,1)$ for the points of this constellation, equivalently $\left(v_{1}, v_{2}, v_{3}, v_{4}, v_{5}\right)=(3,5,4,6,9)$. By the linear proximity inequalities it follows that the cluster $\mathcal{A}:=(\mathcal{C}, \underline{m})$ is idealistic. Now let $I_{\mathcal{A}}$ be the ideal generated by the monomials whose exponents are in the associated Newton polyhedron. We find

$$
I_{\mathcal{A}}=\left(x^{6}, y^{3}, z^{4}, x^{3} y, x^{2} y^{2}, y z^{2}, y^{2} z, x^{3} z, x z^{2}, x y z\right) .
$$

The blowing-up of the constellation gives an embedded resolution for a general element of $I_{\mathcal{A}}$, such as for example $h(x, y, z):=x^{6}+y^{3}+z^{4}+x^{3} y+x^{2} y^{2}+$ $y z^{2}+y^{2} z+x^{3} z+x z^{2}-x y z$. 
Example 3 Let us consider the non-complete ideal $I=\left(x^{3}, y^{2}, z^{2}, x z, x^{2} y\right)$. This ideal is finitely supported and the associated cluster is the idealistic cluster $\mathcal{A}$ from Example 1. Theorem 1 says then that the blowing up of that constellation gives an embedded resolution for a general element of $I$, such as for example $x^{3}+y^{2}+z^{2}+x z+x^{2} y$.

(4) We now first recall the notion for a polynomial to be nondegenerate with respect to its Newton polyhedron. Let $f \in \mathbb{C}\left[x_{1}, \cdots, x_{d}\right]$ be a non-constant polynomial vanishing in the origin. Write $\underline{x}^{\underline{k}}:=x_{1}^{k_{1}} \cdots x_{d}^{k_{d}}$ and $f:=\sum_{\underline{k} \in \mathbb{N}^{d}} c_{\underline{k}} \underline{x}^{\underline{k}}$. The support of $f$ is $\operatorname{supp}(f):=\left\{\underline{k} \in \mathbb{N}^{d} \mid c_{\underline{k}} \neq 0\right\}$. The Newton polyhedron $\Gamma$ of $f$ is the convex hull of $\operatorname{supp}(f)+\mathbb{R}_{\geq 0}^{d}$. For a face $\tau$ of $\Gamma$ we write $f_{\tau}:=\sum_{\underline{k} \in \tau} c_{\underline{k}} \underline{x}^{\underline{k}}$. A polynomial $f$ is called nondegenerate with respect to $\Gamma$ if for every compact face $\tau$ of $\Gamma$, the polynomials $f_{\tau}$ and $\partial f_{\tau} / \partial x_{i}$ have no common zeroes in $\left(\mathbb{C}^{*}\right)^{d}$, $1 \leq i \leq d$.

Proposition 2 Every hypersurface that is general with respect to some 3-dimensional toric idealistic cluster is nondegenerate with respect to its Newton polyhedron.

Proof. Let $\mathcal{A}=(\mathcal{C}, \underline{m})$ be a toric idealistic cluster such that $f$ is general with respect to $\mathcal{A}$. Suppose that $f$ is degenerate with respect to $\mathcal{N}(f)$.

Let $\tau$ be a compact face of $\mathcal{N}(f)$ for which there exists a point $p \in\left(\mathbb{C}^{*}\right)^{3}$ such that $f_{\tau}(p)=\partial f_{\tau} / \partial x(p)=\partial f_{\tau} / \partial y(p)=\partial f_{\tau} / \partial z(p)=0$.

If $\tau$ is a facet, then $\tau$ corresponds to some exceptional irreducible component created by the blowing up of the constellation, say to $E_{i}$. More specifically, the strict transform of $f_{\tau}$ is equal to $E_{0} \cap E_{i}$. As $p$ is not an orbit, it follows that there exists a point in which $E_{0} \cap E_{i}$ does not have normal crossings and that is not an orbit. If the dimension of $\tau$ is one and if $\tau$ is the intersection of two compact facets, then analogously we have that there exist two irreducible exceptional components $E_{i}$ and $E_{j}$ such that $E_{0} \cap E_{i} \cap E_{j}$ does not have normal crossings in a point that is not an orbit. Remains the case that $\tau$ is the intersection of a compact facet and a coordinate plane. Suppose that that compact facet corresponds to $E_{i}$ and that the coordinate plane is given by $\{x=0\}$. Again we get that then $E_{0} \cap E_{i}$ does not have normal crossings in a point that is not an orbit. Indeed, if $E_{i}$ has equation $y=0$ in some affine chart, then there is a point $\left(0,0, p_{z}\right)$ with $p_{z} \neq 0$ in which there are no normal crossings.

\section{Conjectures}

Let $f$ be a complex polynomial in $d$ variables and let $\pi: Z \rightarrow \mathbb{C}^{d}$ be an embedded resolution of singularities of $f^{-1}\{0\}$. We write $E_{j}, j \in S$, for the irreducible components of $\pi^{-1}\left(f^{-1}\{0\}\right)$ and we denote by $N_{j}$ and by $\nu_{j}-1$ the multiplicities of $E_{j}$ in the divisor on $Z$ of $f \circ \pi$ and $\pi^{*}\left(d x_{1} \wedge \ldots \wedge d x_{d}\right)$, respectively. The couples $\left(\nu_{j}, N_{j}\right), j \in S$, are called the numerical data of the embedded resolution $(Z, \pi)$. We denote also $E_{j}^{\circ}:=E_{j} \backslash\left(\cup_{i \in S \backslash\{j\}} E_{i}\right)$, for $j \in S$. Let the $E_{j}$, 
$j \in J:=\{1, \cdots, r\} \subset S$, be the exceptional irreducible components of $\pi^{-1}(\{0\})$.

3.1. Monodromy.- We assume that $f(b)=0$. Take $\epsilon>0$ small enough such that the open ball $B_{\epsilon}$ with radius $\epsilon$ around $b$ in $\mathbb{C}^{d}$ intersects the fibre $f^{-1}(0)$ transversally. Then choose $\epsilon \gg \eta>0$ such that for $t$ in the disc $D_{\eta} \subset \mathbb{C}$ around the origin, the fibre $f^{-1}(t)$ intersects $B_{\epsilon}$ transversally. Write $X:=f^{-1}\left(D_{\eta}\right) \cap B_{\epsilon}$, $X_{t}:=f^{-1}(t) \cap B_{\epsilon}$ for $t \in D_{\eta}$ and $D_{\eta}^{*}:=D_{\eta} \backslash\{0\}$ for the pointed disc. Milnor showed that $f_{\left.\right|_{X \backslash X_{0}}}: X \backslash X_{0} \rightarrow D_{\eta}^{*}$ is a locally trivial fibration, see [Mil68]. A fibre $X_{t}$ of this bundle is called Milnor fibre of $f$ at $b$. We will denote it by $F_{b}$. Consider the loop $\gamma$ encircling the origin once counterclockwise. Since $f_{\left.\right|_{X \backslash X_{0}}}$ is a locally trivial fibration, the loop $\gamma$ lifts to a diffeomorphism $h$ of the Milnor fibre $F_{b}$, which is well determined up to homotopy. In this way $\gamma$ induces an automorphism $h^{*}: H^{i}\left(F_{b}, \mathbb{C}\right) \rightarrow H^{i}\left(F_{b}, \mathbb{C}\right), i \geq 0$, that is called the monodromy transformation.

The surfaces for which we will prove the monodromy conjecture have exactly one isolated singularity in the origin. A result of Milnor (see [Mil68]) then says that $H^{i}\left(F_{0}, \mathbb{C}\right)=0$, for $i \neq 0$ and $i \neq d-1$, and $H^{0}\left(F_{0}, \mathbb{C}\right)=\mathbb{C}$ with trivial monodromy action. The formula of A'Campo ([A'C75]) describes the characteristic polynomial of the monodromy action on $H^{d-1}\left(F_{0}, \mathbb{C}\right)$ in terms of an embedded resolution of the hypersurface $f^{-1}(0)$.

We may suppose that $\pi$ is an isomorphism outside the inverse image of the origin.

Theorem 3 (A'Campo) The characteristic polynomial of the monodromy action on $H^{d-1}\left(F_{0}, \mathbb{C}\right)$ is equal to

$$
\left[\frac{\prod_{j=1}^{r}\left(1-t^{N_{j}}\right)^{\chi\left(E_{j}^{\circ}\right)}}{1-t}\right]^{(-1)^{d-1}}
$$

3.2. Topological zeta function.- In 1992 Denef and Loeser created a new zeta function which they called the topological zeta function because of the topological Euler-Poincaré characteristic $\chi(\cdot)$ turning up in it. It is associated to a complex polynomial $f$ with $f(0)=0$. If $E_{I}:=\cap_{i \in I} E_{i}$ and $E_{I}^{\circ}:=E_{I} \backslash$ $\left(\cup_{j \notin I} E_{j}\right)$, then they introduced it in [De,L92] in the following way.

Definition 4 The local topological zeta function associated to $f$ is the rational function in one complex variable

$$
Z_{t o p, f}(s):=\sum_{I \subset S} \chi\left(E_{I}^{\circ} \cap \pi^{-1}\{0\}\right) \prod_{i \in I} \frac{1}{N_{i} s+\nu_{i}} .
$$

Denef and Loeser proved that every embedded resolution gives rise to the same function, so the topological zeta function is a well-defined singularity invariant (see [De,L92]). Once the motivic Igusa zeta function was introduced, they proved this result alternatively in [De,L98] by showing that this more general zeta function specialises to the topological one. There exists a global version, 
replacing $E_{I}^{\circ} \cap \pi^{-1}\{0\}$ by $E_{I}^{\circ}$.

3.3. Monodromy conjecture.- One calls $\alpha$ an eigenvalue of monodromy of $f$ at $b \in f^{-1}\{0\}$ if $\alpha$ is an eigenvalue for some $h^{*}: H^{i}\left(F_{b}, \mathbb{C}\right) \rightarrow H^{i}\left(F_{b}, \mathbb{C}\right)$.

Conjecture 5 (Monodromy Conjecture) If $s_{0}$ is a pole of $Z_{\text {top, } f}$, then $e^{2 \pi i s_{0}}$ is an eigenvalue of monodromy of $f$ at some point of the germ at 0 of the hypersurface $f=0$.

Let $f$ be a polynomial that is general with respect to a 3-dimensional toric idealistic cluster. Consider the embedded resolution $\pi: Z \rightarrow \mathbb{C}^{3}$ of $f^{-1}\{0\}$ that corresponds to the blowing up of the constellation. We fix a candidate pole $s_{0}=-\nu_{j} / N_{j}$ of $Z_{t o p, f}$. If $E_{j}$ is not an exceptional component, then $\nu_{1}=1$ and $N_{1}=1$. As 1 is always an eigenvalue of the local monodromy of $f$, this candidate pole does not pose any difficulty. If $s_{0}=-\nu_{j} / N_{j}$ is a candidate pole of $Z_{t o p, f}$ induced by an exceptional component $E_{j}$, then we write $\nu_{j} / N_{j}$ as $a / b$ such that $a$ and $b$ are coprime. We define the set $J_{b}:=\left\{j \in J \mid b\right.$ divides $\left.N_{j}\right\}$. It follows from A'Campo's formula that

$$
\begin{aligned}
& e^{2 \pi i s_{0}} \text { is an eigenvalue of monodromy of } f \text { at the origin } 0 \\
& \qquad \sum_{j \in J_{b}} \chi\left(E_{j}^{\circ}\right) \neq 0 .
\end{aligned}
$$

In general there can be a lot of cancelations which make that $\sum_{j \in J_{b}} \chi\left(E_{j}^{\circ}\right)=0$. To control this, we will determine when $\chi\left(E_{j}^{\circ}\right)$ is positive, negative or zero. We will see that the cases where $\chi\left(E_{j}^{\circ}\right) \leq 0$ are very rare in this context.

3.4. Holomorphy conjecture.- For every $r \in \mathbb{Z}_{>0}$, one can define a variant $Z_{t o p, f}^{(r)}$ of the topogical zeta function that is also a rational function in one complex variable.

\section{Definition 6}

$$
Z_{t o p, f}^{(r)}:=\sum_{\substack{I \subset S \\ \forall i \in I: r \mid N_{i}}} \chi\left(E_{I}^{\circ} \cap \pi^{-1}\{0\}\right) \prod_{i \in I} \frac{1}{N_{i} s+\nu_{i}} .
$$

The functions $Z_{t o p, f}^{(r)}$ are limits of more general Igusa zeta function associated to a polynomial and a character (see [De91]). In particular $Z_{t o p, f}^{(1)}=Z_{t o p, f}$. Clearly they are holomorphic on $\mathbb{C}$ if and only if they do not have a pole. The holomorphy conjecture stated by Denef predicts the following relation.

Conjecture 7 (Holomorphy conjecture) If $r \in \mathbb{Z}_{>0}$ does not divide the order of any eigenvalue of monodromy of $f$, then $Z_{\text {top }, f}^{(r)}$ is holomorphic on $\mathbb{C}$.

In Section 9 we provide a proof of the holomorphy conjecture for the surfaces we are studying. Again, the classification of $\chi\left(E_{j}^{\circ}\right)$ according to the sign will be the key to solve the conjecture. 